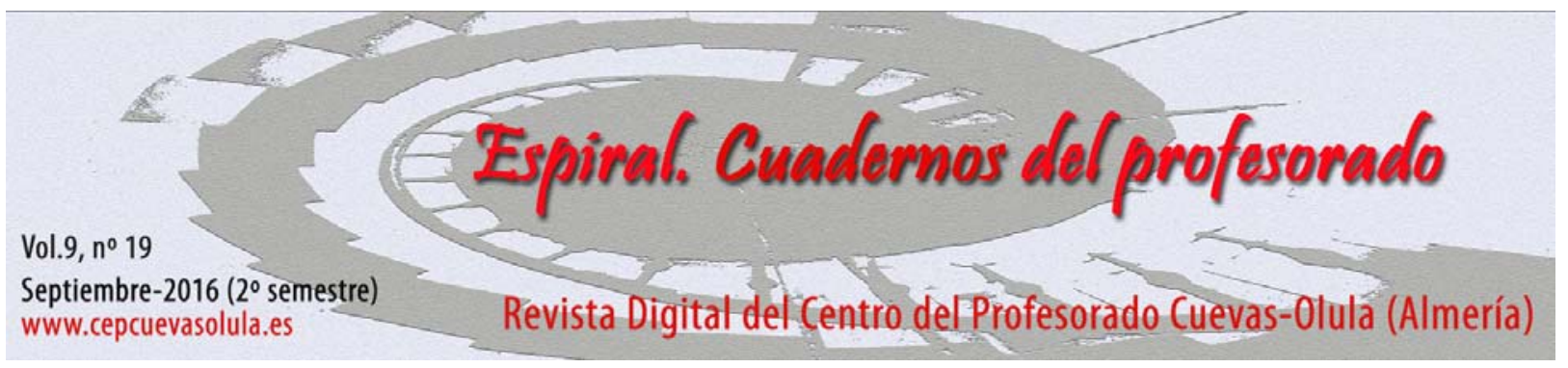

\title{
PROPUESTA DOCENTE PARA LA ASIGNATURA ANÁLISIS DE DATOS DEL GRADO EN CIENCIAS DE LA ACTIVIDAD FÍSICA Y DEL DEPORTE
}

Teaching proposal for data analysis subject of physical activity and sport sciences degree

\section{María Carrasco Poyatos}

\author{
Universidad Católica San Antonio de Murcia, España
}

\begin{abstract}
RESUMEN: La guía docente constituye una herramienta básica para alcanzar los principios que guían el proceso de Convergencia en la creación del Espacio Europeo de Educación Superior (EEES). La planificación de la asignatura debe situarse sobre un doble eje: el contenido y el trabajo del estudiante en base a ese contenido. Por tanto, una guía docente debe especificar la selección y ordenación de contenidos académicos, cómo se trabajaran dichos contenidos y la forma de evaluación de los estudiantes. La propuesta que se presenta pretende dar unas orientaciones sobre las competencias a adquirir por parte de los estudiantes, la estructura de los contenidos de la materia, qué enseñar y aprender a partir de los contenidos, el desarrollo didáctico para alcanzar los aprendizajes, la dedicación del estudiante y los procedimientos de evaluación y calificación del aprendizaje, para la asignatura Análisis de Datos del Grado en Ciencias de la Actividad Física y del Deporte perteneciente a la Universidad Católica San Antonio de Murcia (UCAM), para favorecer el proceso de enseñanza-aprendizaje de los estudiantes.
\end{abstract}

Palabras clave: Propuesta docente, Espacio Europeo de Educación Superior, Convergencia, planificación, Análisis de Datos.

ABSTRACT: The teaching guide is a basic tool to reach the Convergence process guiding principles in the European Space of Higher Education (EEES). Each subject design should follow a double way: the content and the student work related to this content. Therefore, a teaching guide should specify the academic content selection and ordination, how will these contents be worked out, and the students evaluation method. Our teaching guide is an approach about the competences students should acquire, the subject content structure, what to teach and learn from these contents, the educational development to reach the learning, the student dedication and the learning evaluation and score methods, in the subject Data Analysis from the Degree in Sciences of Physical Activity and Sports of the San Antonio Catholic University of Murcia (UCAM), to advantage the students teaching-learning process.

Key words: teaching proposal, European Space of Higher Education, Convergence, planning, Data Analysis.

Carrasco Poyatos, M. (2016). Propuesta docente para la asignatura Análisis de Datos del grado en Ciencias de la Actividad Física y del Deporte. Espiral. Cuadernos del Profesorado, 9(19), 78-87. Disponible en: http://espiral.cepcuevasolula.es/

Fecha de recepción: 13/04/2016

Fecha de aceptación: 15/07/2016
Enviar correspondencia a: mcarrasco@ucam.edu 


\section{Introducción}

Las universidades europeas de los distintos países han aceptado el reto de adoptar las principales líneas de actuación que indica el nuevo Espacio Europeo de Educación Superior (EEES). Para llevar a cabo este proceso de reforma se cambió tanto en la estructura como en el contenido del sistema educativo universitario, que se constituyó sobre el Sistema Europeo de Transferencia de Créditos (ECTS o European Credit Transfer System) y el suplemento europeo al título. Los créditos ECTS constituyen un sistema centrado en el alumno, que se basa en la carga de trabajo del estudiante necesaria para la consecución de los objetivos de un programa (ANECA, 2003). Es, por tanto, una medida del trabajo total del alumno expresada en horas, que incluye tanto las clases presenciales (teóricas y prácticas) como el esfuerzo dedicado al estudio, a los trabajos y a la preparación y realización de exámenes.

Las líneas de actuación del EEES sugirieron la renovación del diseño de los planes de estudio y las programaciones docentes, teniendo como referencia el aprendizaje o la adquisición de competencias de los alumnos, que a su vez tienen que participar de manera más activa en su propia formación. Esto conlleva que tanto la planificación como la realización de los procesos de enseñanzaaprendizaje se lleven a cabo asumiendo este punto de vista (De Miguel, 2006). De ahí que el denominado "cambio de paradigma del proceso enseñanza-aprendizaje" se estableciera como uno de los objetivos prioritarios a tener en cuenta en el diseño de los planes de estudios. La nueva visión del proceso de enseñanza-aprendizaje implica establecer una buena planificación para el desarrollo de la docencia, con el fin de facilitar el aprendizaje de los estudiantes y, como consecuencia, caminar hacia la mejora de la calidad del propio proceso (Díez, Pacheco, y García, 2008).

Para ello, los docentes han tenido que transformarse en guías del proceso de aprendizaje de los alumnos (Zabalza, 2004). La planificación didáctica de una materia no puede limitarse a distribuir los contenidos a lo largo de un cronograma utilizando como sistema de cómputo de la actividad docente los créditos europeos, sino exponer secuencialmente todo el conjunto de actividades y tareas a realizar para tutorizar las experiencias de aprendizaje de los estudiantes (De Miguel, 2006). Por tanto, el proceso englobará desde la definición de las competencias de la titulación hasta el diseño de los procedimientos de evaluación, que se deben definir paralela e integradamente en relación a las competencias a alcanzar (Biggs, 2005; Prieto, 2004). La planificación, por tanto, va a obligar al docente a organizarse en función del periodo lectivo y a reflexionar sobre qué va a hacer y para qué va a hacerlo.

El reto es que un estudiante medio pueda conseguir las competencias que se proponen como metas de aprendizaje, para lo que se ha hecho indispensable (De Miguel, 2006; Vizcarro, 2008): una actividad académica centrada en el empleo de metodologías que enfaticen el autoaprendizaje, el trabajo guiado, el aprendizaje cooperativo y el acercamiento a la realidad laboral del alumno; el trabajo de competencias de carácter transversal como la iniciativa, el sentido crítico, el trabajo en equipo, la redacción de informes, la exposición en público, etc; la tendencia a la interdisciplinariedad o interconexión entre asignaturas, y el incremento del peso de valoraciones periódicas que conformen una evaluación continuada.

A su vez, nos encontramos envueltos en una sociedad caracterizada por el uso y avance de las nuevas tecnologías de la información y la comunicación (TIC) (Carrasco, Abraldes, y Gómez, 2012). Si se pretende conseguir un sistema educativo de calidad, es fundamental que en la metodología docente universitaria éstas se vean incluidas. A través de las TIC se pueden alcanzar las siguientes competencias: búsqueda y selección de información, análisis crítico y resolución de problemas, trabajo en equipo, idiomas, capacidad de autoaprendizaje y de adaptación al cambio, interdisciplinariedad o iniciativa, y perseverancia (Medaña y González, 2004; Huertas y Tenorio, 2006). Por tanto, aunque el uso de las TIC no garantice por sí mismo el éxito en el proceso de enseñanza-aprendizaje, sí que favorece un aprendizaje más personalizado y autónomo, basado en los conocimientos previos de los estudiantes, y facilitando la posibilidad de solicitar y recibir asesoramiento por parte de los profesores y los compañeros. 
Para que todo esto surta el efecto deseado, debe llevarse a cabo en base a un equilibrio entre las exigencias que imponen las titulaciones y la propia organización del curso académico y los medios con los que cuentan los estudiantes para dar respuesta a estas exigencias (Sánchez-Bascones, RuizEsteban, y Pascual-Gómez, 2011). La guía docente será una buena herramienta para planificar las materias, ubicándolas en el contexto de la titulación a la que pertenecen.

\section{Las guías docentes en el marco del EEES}

Existen tres tipos de guías en el sistema educativo universitario, que son inclusivas unas en otras. En primer lugar está la guía de la titulación, que es un marco informativo de las materias y asignaturas propias de la titulación. Incluida en ésta, la guía docente pretende informar y orientar a los estudiantes en el estudio de cada una de las materias, desde la perspectiva del profesor. Y por último, la guía didáctica debe detallar los aprendizajes que se espera que logren los alumnos, y los procedimientos y medios necesarios para alcanzarlos.

La guía docente es un documento didáctico elaborado con el objetivo de informar y orientar a los estudiantes en su estudio y constituye una herramienta para el desarrollo de uno de los principios básicos de la convergencia: "docencia basada en el aprendizaje" (Zabalza, 2004). Aunque la responsabilidad sobre la elaboración de las guías docentes es compartida por los centros y los departamentos universitarios, finalmente es cada profesor quien debe asumir que su implicación es crucial para que éstas puedan ser realmente útiles y puedan incidir en la modificación de los sistemas de trabajo del estudiante (Sánchez-Bascones et al., 2011).

Debido a que en el sistema educativo universitario las asignaturas están organizadas por módulos, las directrices del EEES requieren el fortalecimiento de las acciones de coordinación dentro de las titulaciones, por lo que debe abandonarse la idea de materia como espacio científico independiente y desconectado del resto de materias, cobrando importancia el contexto donde se ubica (Sánchez-Bascones et al., 2011), procurando la no coincidencia de contenidos con otras materias.

Seguir la misma estructura de guía docente en todas las materias de la titulación, facilita la coordinación entre ellas y su comprensión por parte del estudiante. Varios autores proponen como elementos imprescindibles de la guía docente (Zabalza, 2004; Vizcarro, 2007, Sánchez-Bascones et al., 2011):

I. La justificación de la materia: qué aporta la asignatura al plan de estudios; su relación con otras, con el plan de estudios y con la profesión; y conocimientos previos necesarios.

II. La descripción de la materia: resultados esperados de aprendizaje, conocimientos y competencias; y contenidos.

III. Organización de la materia: actividades de aprendizaje, sistema de evaluación, estructuración temporal, y recursos.

A continuación se detalla una propuesta docente para la asignatura Análisis de Datos del Grado en Ciencias de la Actividad Física y del Deporte de la UCAM, siguiendo las indicaciones de los autores anteriormente citados, para homogeneizar la estructura y facilitar su adaptación a otras asignaturas similares.

\section{Propuesta docente de la asignatura Análisis de Datos del Grado en Ciencias de la Actividad Física y del Deporte}

Siguiendo la estructura que recomiendan Sánchez-Bascones et al. (2011) y las orientaciones didácticas de De Miguel (2006) y Vizcarro (2007), se detalla a continuación la propuesta docente de la asignatura Análisis de Datos del Grado en Ciencias de la Actividad Física y del Deporte de la UCAM:

\section{Datos descriptivos de la materia y de su docencia.}


Dentro del Grado en Ciencias de la Actividad Física y del Deporte de la Universidad Católica San Antonio de Murcia (UCAM), la asignatura Análisis de Datos se engloba dentro del Módulo Ciencias Aplicadas y Motricidad, y la Materia Ciencias Aplicadas a la Actividad Física y al Deporte, junto con las asignaturas: Fundamentos Conceptuales de las Ciencias del Deporte, Metodología de la Investigación en Actividad Física y Deporte, Técnicas, Test e Instrumentos de Registro, y Nuevas Tecnologías en Actividad Física y Deporte. Es un tipo de asignatura de Formación Básica, con 6 créditos ECTS, cuatrimestral, que se imparte en $2^{\circ}$ curso.

\section{Sentido de la materia en el Plan de Estudios.}

La asignatura de Análisis de Datos está centrada en el desarrollo de herramientas relacionadas con el manejo y tratamiento de conjuntos de datos. Dentro del proceso de investigación, esta asignatura consiste en la primera incursión al análisis de datos que forma parte de dicho proceso. Sería paso siguiente a la asignatura Fundamentos Conceptuales de las Ciencias del Deporte, que se imparte en $1^{\circ}$ curso, y que se centra más en la primera fase del proceso de investigación (revisión bibliográfica, identificar las variables y el diseño de estudio); y el paso previo a la asignatura Metodología de la Investigación en Actividad Física y Deporte, que se imparte en $4^{\circ}$ curso, y que profundiza en el análisis estadístico. Una vez adquiridas las competencias propuestas, el alumno también podrá poner en práctica sus conocimientos en las asignaturas Técnicas, Test e Instrumentos de Registro, y Nuevas Tecnologías en Actividad Física y Deporte.

Para hacerla específica de las Ciencias de la Actividad Física y del Deporte, se usarán bases de datos de los perfiles profesionales propios de las Ciencias del Deporte: gestión, educación, salud, recreación y rendimiento. Se debe aprender a dominar tanto los procesos de adquisición de datos, como la forma más adecuada de tratarlos para extraer de ellos información útil que permita tomar decisiones correctas. Todo ello tomando como base los procedimientos estadísticos pero sin perder de vista la aplicación práctica de los mismos. Dado que hoy en día el manejo de las TIC se hace imprescindible en cualquier ámbito laboral, se trata de que el alumno adquiera las competencias necesarias para desenvolverse en su futuro ámbito laboral con la destreza y calidad propias de su titulación.

No se necesitan conocimientos previos específicos para cursarla.

3. Competencias a desarrollar y su concreción en resultados de aprendizaje.

$>$ Competencias transversales:

- (CT1) Capacidad de análisis y síntesis.

- (CT2) Capacidad de organización y planificación.

- (CT3) Comunicación oral y escrita en lengua nativa.

- (CT5) Conocimientos de informática relativos al ámbito de estudio.

- (CT6) Capacidad de gestión de la información.

- (CT7) Resolución de problemas.

- (CT8) Toma de decisiones.

- (CT9) Trabajo en equipo.

- (CT11) Habilidad en relaciones interpersonales.

- (CT13) Razonamiento crítico.

- (CT14) Compromiso ético.

- (CT15) Aprendizaje autónomo.

- (CT21) Motivación por la calidad.

Competencias específicas:

- (CSE8) Aplicar las herramientas estadísticas básicas en el ámbito de las Ciencias de la Actividad Física y del Deporte.

Competencias UCAM: 
- (CUCAMT1) Ser capaz de expresarse correctamente en castellano en su ámbito disciplinar.

- (CUCAMT3) Ser capaz de proyectar los conocimientos, habilidades y destrezas adquiridos para promover una sociedad basada en los valores de la libertad, la justicia, la igualdad y el pluralismo.

- (CUCAMT5) Ser capaz de utilizar como usuario las herramientas básicas en TIC.

- (CUCAMT6) Capacidad para trabajar en equipo, relacionándose con otras personas del mismo o distinto ámbito profesional.

- (CUCAMT7) Desarrollar habilidades de iniciación a la investigación.

Resultados de aprendizaje:

- Conocer y utilizar las posibilidades que la informática, en sus diferentes aplicaciones, ofrece a su ámbito de estudio.

- Comprender y distinguir las características que tienen las distintas herramientas de análisis de datos.

- Interpretar los análisis estadísticos básicos de la literatura científica específica de la actividad física y el deporte.

- Determinar y utilizar las herramientas estadísticas más adecuadas para el análisis de datos de estudios de investigación en la actividad física y el deporte para el desempeño de sus actividades formativas y profesionales.

- Comprender, razonar y sintetizar contenidos de diversos ámbitos de conocimiento.

- Gestionar y organizar la información adquirida durante el proceso de aprendizaje.

- Expresarse correctamente de forma oral y por escrito en su lengua nativa.

- Organizar y saber utilizar la información procedente de diferentes contextos.

- Decidir, de manera integral y crítica, entre diferentes opciones.

- Adquirir e implementar estrategias de colaboración y habilidades que favorezcan el trabajo cooperativo.

- Desarrollar su propia labor profesional en un entorno globalizado.

- Adquirir y poner en práctica habilidades sociales y comunicativas que favorezcan la interacción.

- Gestionar de manera proactiva su proceso de aprendizaje.

- Conocer y usar adecuadamente los recursos que posibilitan las nuevas tecnologías de la información y la comunicación.

- Emprender acciones que fomenten el interés y la motivación por la investigación.

4. Contenidos.

I. Tema 1. Introducción.

- Competencias: CT1, CT3, CT6, CT13, CUCAMT1.

- Cronograma: 2 sesiones (S1-2). Total: $4 \mathrm{~h}$.

- Contenidos teóricos:

I.1. Presentación.

I.2. Definición: dato y análisis de datos.

I.3. Tipos de estudios.

I.4. El proceso básico de análisis de datos en el ámbito de las Ciencias del Deporte.

II. Tema 2. Definir: diseño previo al análisis.

- Competencias: CT1, CT2, CT3, CT6, CT7, CT8, CT9, CT13, CT21, CUCAMT1, CUCAMT5, CUCAMT7. 
- Cronograma: 2 sesiones (S3-4). Total: $4 \mathrm{~h}$.

- Contenidos teóricos:

II.1. Objetivo del estudio.

II.1.1. Planteamiento de una pregunta/problema a resolver.

II.1.2. Revisar evidencias sobre el tema.

II.1.3. Delimitar el objetivo.

II.2. Elección de la muestra.

II.2.1. Definir la población de estudio.

II.2.2. Especificar los criterios de selección.

II.2.3. Estimar el tamaño de la muestra.

II.3. Apoyo al temario teórico.

Práctica 1 (T2_p1). Determinar el objetivo de estudio y seleccionar la muestra.

III. Tema 3. Dividir: preparar los datos.

- Competencias: CT3, CT5, CT7, CT8, CT9, CT11, CT13, CT14, CT15, CT21, CSE8, CUCAMT1, CUCAMT3, CUCAMT5, CUCAMT6, CUCAMT7.

- Cronograma: 8 sesiones (S5- S12). Total: $16 \mathrm{~h}$.

- Contenidos teóricos:

III.1. Recogida de datos.

III.1.1. Selección de las variables.

III.1.2. Definición de las variables.

III.1.3. Carácter de las variables.

III.1.4. Tipo de variables.

III.2. Preparar los datos.

III.3. Apoyo al temario teórico.

Práctica 2 (T3_p1). Recogida y preparación de datos: búsqueda en la web, tipos de pegado, definición de variables, formato de celdas: fuente, ordenar y filtrar.

Práctica 3 (T3_p2). Recogida y preparación de datos: búsqueda en la web, tipos de pegado, definición de variables, formato de celdas: alineación y celdas, formato condicional.

Práctica 4 (T3_p3). Recogida y preparación de datos: definición de variables, texto en columnas, buscar datos, codificación de variables, función contar.

Práctica 5 (T3_p4). Recogida y preparación de datos: formulación y \$.

- Contenidos prácticos:

Seminario 1. Seminario trabajo grupal (I).

Taller 1. Diseño previo al análisis y preparación de datos.

Taller 2. Formulación.

IV. Tema 4. Evaluar: analizar los datos.

- Competencias: CT3, CT5, CT7, CT8, CT9, CT11, CT13, CT14, CT15, CT21, CSE8, CUCAMT1, CUCAMT3, CUCAMT5, CUCAMT6, CUCAMT7.

- Cronograma: 14 sesiones (S13-26). Total: 28h.

- Contenidos teóricos:

IV.1. Análisis descriptivo. 
IV.1.1. Estadísticos para variables cualitativas: distribución de frecuencias.

IV.1.2. Estadísticos para variables cuantitativas: centralización, dispersión, y forma.

IV.2. Análisis inferencial.

IV.2.1. Introducción al cálculo de probabilidades.

IV.2.2. Factores de interacción y factores de confusión.

IV.3. Apoyo al temario teórico.

Práctica 6 (T4_p1). Análisis descriptivo para toda la muestra. Variables cualitativas. Tablas y gráficos.

Práctica 7 (T4_p2). Análisis descriptivo para toda la muestra. Variables cualitativas y cuantitativas (estadísticos de centralización). Tablas y gráficos.

Práctica 8 (T4_p3). Análisis descriptivo para muestra estratificada. Variables cualitativas y cuantitativas (estadísticos de centralización). Tablas y gráficos.

Práctica 9 (T4_p4). Análisis descriptivo para muestra estratificada. Variables cualitativas y cuantitativas (estadísticos de centralización). Tablas y gráficos dinámicos.

Práctica 10 (T4_p5). Análisis descriptivo. Medidas de dispersión y forma. Tablas y gráficos.

Práctica 11 (T4_p6). Introducción al cálculo de probabilidades.

Práctica 12 (T4_p7). Factor de interacción y confusión.

- Contenidos prácticos:

Seminario 2. Seminario trabajo grupal (II).

Taller 3. Análisis descriptivo de variables cualitativas y cuantitativas (estadísticos de centralización) para toda la muestra. Tablas y gráficos.

Taller 4. Análisis descriptivo de variables cualitativas y cuantitativas (estadísticos de centralización) para la muestra estratificada. Tablas y gráficos dinámicos.

Taller 5. Análisis descriptivo de variables cuantitativas. Estadísticos de dispersión y forma.

Taller 6. Probabilidades. Factor de interacción y confusión.

- Otras modalidades organizativas:

Tutoría 1.

Evaluación 1.

V. Tema 5. Decidir: sacar conclusiones.

- Competencias: CT1, CT2, CT3, CT5, CT8, CT9, CT11, CT14, CT21, CSE8, CUCAMT1, CUCAMT3, CUCAMT5, CUCAMT6, CUCAMT7.

- Cronograma: 4 sesiones (S27-S30). Total: 8h.

- Contenidos teóricos:

V.1. Generación de informes concluyentes.

V.2. Otros programas compatibles.

V.3. Apoyo al temario teórico.

Práctica 13 (T5_p1). Informes (I).

- Contenidos prácticos:

Seminario 3. Seminario trabajo grupal (Exposición).

Taller 7. Generación de informes. 
- Otras modalidades organizativas:

Tutoría 2.

Evaluación 2.

5. Metodología y recursos disponibles.

$>$ Método de enseñanza: lección magistral. El profesor transmite conocimientos y activa procesos cognitivos en el estudiante.

- Se empleará en: la enseñanza de los contenidos teóricos.

- Materiales necesarios: ordenador, presentación power point y/o documento Excel, conexión a internet.

- Carácter: obligatorio y presencial.

Método de enseñanza: resolución de ejercicios y problemas. El alumno ensaya y pone en práctica los conocimientos previos transmitidos en la lección magistral.

- Se empleará en: la enseñanza de los contenidos teóricos, en la parte de apoyo a los contenidos teóricos. El profesor le facilitará documento Excel con la tarea a realizar. También se empleará para la realización de los talleres.

- Materiales necesarios: ordenador, documento Excel, conexión a internet.

- Carácter: obligatorio y presencial.

Método de enseñanza: tutoría entre iguales. Un alumno más aventajado enseña/ayuda a otro que lo es menos, bajo la supervisión del profesor.

- Se empleará en: la enseñanza de los contenidos teóricos, en la parte de apoyo a los contenidos teóricos. El profesor le facilitará documento Excel con la tarea a realizar.

- Materiales necesarios: ordenador, documento Excel, conexión a internet.

- Carácter: obligatorio y presencial.

Método de enseñanza: tutoría académica. El profesor guía y orienta al estudiante en múltiples aspectos académicos ajustando la enseñanza a sus características personales.

- Se empleará en: la enseñanza de los contenidos teóricos, en las sesiones dedicadas a resolver dudas de cara a la evaluación.

- Materiales necesarios: ordenador, presentación power point y/o documento Excel, conexión a internet.

- Carácter: obligatorio y presencial.

Método de enseñanza: seminario. Enseñanza de trabajo en pequeños grupos.

- Se empleará en: la enseñanza de los contenidos prácticos, en las sesiones dedicadas a guiar a los estudiantes en la realización del trabajo grupal.

- Materiales necesarios: ordenador, documento Excel, conexión a internet.

- Carácter: obligatorio y presencial.

Método de enseñanza: aprendizaje cooperativo. Los estudiantes, en grupos reducidos, colaboran en la consecución de los objetivos de aprendizaje a partir de una propuesta de trabajo.

- Se empleará en: la enseñanza de los contenidos prácticos, para la realización del trabajo grupal.

- Materiales necesarios: ordenador, documento Excel, conexión a internet.

- Carácter: obligatorio y no presencial.

6. Cronograma y distribución de las cargas de trabajo en ECTS.

Siguiendo las directrices de ANECA (2003), cada crédito ECTS corresponderá con 25 horas de trabajo del alumno, de las que el $40 \%$ serán presenciales y/o de interacción con el profesor, y el 
$60 \%$, de trabajo autónomo. Por lo que los créditos de la asignatura Análisis de Datos se repartirán en 60 horas presenciales y 90 de trabajo autónomo. La distribución de las cargas de trabajo para cada recurso metodológico se muestra en la Tabla 1.

\section{Sistema de evaluación. Instrumentos y criterios.}

El sistema de evaluación se basará en el paradigma de evaluación continua y formativa, mediante el que las actividades de evaluación sumativas y finales se complementarán con actividades de evaluación formativa y continua. Los procedimientos y técnicas evaluativas serán:

$>$ Pruebas objetivas de elección múltiple. Se utilizarán para evaluar los contenidos teóricos. Constarán de dos pruebas de evaluación parciales. Cada una de ellas supondrá un 30\% de la nota final de la asignatura. Si el alumno supera la prueba, eliminará esa parte de la material. Ambas son obligatorias para superar la asignatura.

$>$ Trabajos y proyectos. Se utilizará para evaluar el trabajo grupal, que forma parte de la parte práctica de la asignatura. Este deberá realizarse de forma autónoma por los estudiantes, organizados en pequeños grupos. Supondrá un $20 \%$ de la nota final de la asignatura. Es obligatorio superarlo para aprobar la asignatura.

$>$ Pruebas de ejecución de tareas reales o simuladas. Se utilizará para evaluar los talleres, que forma parte de la parte práctica de la asignatura. Estos consisten en pruebas en las que el estudiante, de forma autónoma e individual, debe demostrar que ha adquirido las competencias necesarias para conseguir resolver un problema. Se plantearán a medida que avanza la materia y se corregirán durante el curso para que el estudiante reciba un feedback sobre la evolución de su proceso de aprendizaje. Supondrán un $20 \%$ de la nota final de la asignatura. No será obligatorio entregarlos. Tendrán carácter sumativo.

\section{Bibliografía.}

\section{Bibliografía básica}

- Banfield C (2011). Excel 2010 para Dummies. Madrid: CEAC. (Temas 2, 3 y 4)

- Burgos R. (1998). Metodología de investigación y escritura científica en clínica. Granada: Escuela Andaluza de Salud Pública. (Tema 4)

- Prieto, L., y Herranz, I. (2012). Bioestadística sin dificultades matemáticas. Ed. Díaz de Santos. (Tema 4).

- Thomas, J., y Nelson, J. (2006). Métodos de investigación en actividad física. Barcelona: Paidotribo. (Tema 4).

Bibliografía complementaria:

- Herranz, I., y Prieto, L. (2004). ¿Qué significa “estadísticamente significativo”? La falacia del criterio del 5\% en la investigación científica. Madrid: Díaz de Santos. (Tema 4)

- Meyer, E. M. (2007). Manual de Excel. En: http://aplicaexcel.galeon.com/Demos/DM_ManualExcel.pdf(Temas 2, 3 y 4)

Webs relacionadas:

- http://www.ucam.edu/biblioteca/biblioteca-digital/acceso-ucam

- http://www.the-sports.org

- http://www.ine.es

- http://www.google.drive.es

- http://www.scholar.google.com

- http://www.aulaclic.es/excel2010 


\section{Conclusiones}

Las líneas de actuación que propone el EEES engloban una nueva visión del proceso de enseñanza-aprendizaje que requiere una buena planificación para el desarrollo de la docencia. Esto supone al docente la compleja tarea de reorganizar el conjunto de actividades y tareas, incluyendo el uso de las TIC, para que el estudiante pueda conseguir las competencias que se proponen como metas de aprendizaje. La propuesta que se presenta pretende ser una herramienta para ayudar a organizar la docencia en el marco del EEES. Ésta se viene poniendo en práctica durante varios cursos académicos en el Grado de Ciencias del Deporte de la UCAM, con excelentes resultados en cuanto a la evaluación de la materia por parte de los alumnos.

\section{Referencias}

ANECA. (2003). Programa de Convergencia Europea. El Crédito Europeo [En Línea]. Disponible en: http://www.aneca.es/publicaciones/docs/publi_credito\%20europeo.pdf. Consulta [07/07/2016].

Biggs, J. (2005). Calidad del aprendizaje universitario. Madrid: Narcea.

Carrasco, M., Abraldes, A., y Gómez, M. (2012). Utilización de las TIC como recurso metodológico. Propuesta de una webquest como aplicación práctica en actividad física y deporte. Actividad Física y Deporte: Ciencia y Profesión, 16, 13-20.

De Miguel, M. (2006). Modalidades de enseñanza centradas en el desarrollo de competencias. Orientaciones para promover el cambio metodológico en el Espacio Europeo de Educación Superior. Oviedo: Ediciones Universidad de Oviedo.

Díez, C., Pacheco, D.I., y García, J.N. (2008). Las habilidades sociales en el marco del EEES. En, M. Hijano del Río, Las titulaciones de educación ante el Espacio Europeo de Educación Superior: análisis de experiencias (pp. 239-247). Archidona (Málaga): Aljibe.

Huertas, J.M., y Tenorio, A.F. (2006). Nuevas tecnologías en la didáctica de la estadística: WebQuest. Rect@, 1, 27. XIV Jornadas de ASEPUMA y II Encuentro Internacional. Universidad Pablo de Olavide [En Línea]. Disponible en: http://www.uv.es/asepuma/XIV/comunica/113NUEVA.pdf. Consulta [07/07/2016].

Mendaña, C.,n y González, B. (2004). El papel de las WebQuest como herramienta para el aprendizaje del alumno en la nueva sociedad del conocimiento. Actas Virtuales del III Simposio Virtual de Computación en la Educación. [En Línea]. Disponible en: http://www.somece.org.mx/virtual2004/ponencias/contenidos/CuervoCristia.htm. Consulta [07/07/2016].

Prieto, L. (2004). La alineación constructiva en el aprendizaje universitario. En, J. C. Torre y E. Gil (Eds), Hacia una enseñanza universitaria centrada en el aprendizaje. Libro homenaje a Pedro Morales Vallejo. Madrid: Universidad Pontificia de Comillas.

Sánchez-Bascones, M., Ruiz-Esteban, C., y Pascual-Gómez, I. (2011). La guía docente como eje del proceso de enseñanza-aprendizaje. Bordón, 63(2), 53-64.

Vizcarro, C. (2007) Algunas estrategias docentes. Unidad de Innovación y Calidad Educativas: Universidad de Castilla-La Mancha.

Vizcarro, C. (2008). Ideas para enseñar en ECTS [En línea]. Disponible en: https://www.uclm.es/organos/vic_docencia/uie/pdf/planificacion/CIDEAS.pdf. Consulta [07/07/2016].

Zabalza, M. A. (2004). Guía para la planificación didáctica de la docencia universitaria en el marco del EEES (Guía de guías) [En línea]. Disponible en: http://www.udc.es/grupos/apumefyr/docs/guiadeguias.pdf. Consulta [07/07/2016]. 